\title{
Ion resonance acceleration by dipolarization fronts: analytic theory and spacecraft observation
}

\author{
A. V. Artemyev ${ }^{1,2}$, V. N. Lutsenko ${ }^{1}$, and A. A. Petrukovich ${ }^{1}$ \\ ${ }^{1}$ Space Research Institute, RAS, Moscow, Russia \\ ${ }^{2}$ UMR6115, LPC2E/CNRS - University of Orleans, Orleans, France \\ Correspondence to: A. V. Artemyev (artemyev@iki.rssi.ru)
}

Received: 7 September 2011 - Revised: 28 November 2011 - Accepted: 3 January 2012 - Published: 7 February 2012

\begin{abstract}
In this paper, we consider the mechanism of ion acceleration by dipolarization fronts in the Earth's magnetotail. The statistics of dipolarization front observations by Interball-tail have been collected from 1995 to 1998 (51 events). We demonstrate that near dipolarization fronts bursts of energetic ions are often observed with an average energy of about $100-200 \mathrm{keV}$. We develop the analytical model of the ion resonance interaction with dipolarization fronts to describe the observed acceleration. We compare the model and the observations to estimate the width of fronts along the dawn-dusk direction, $R_{\mathrm{y}}$. The mean value is $\left\langle R_{\mathrm{y}}\right\rangle \sim 6 R_{\mathrm{E}}$.
\end{abstract}

Keywords. Magnetospheric physics (Magnetotail) - Space plasma physics (Charged particle motion and acceleration; Wave-particle interactions)

\section{Introduction}

During the last decade, multispacecraft missions (Cluster and THEMIS) reveal various mesoscale (a few $R_{\mathrm{E}}$ ) transient structures in the Earth's magnetotail (see review by Sharma et al., 2008). These structures can be understood as spatially localized nonlinear current systems. The magnetic field of mesoscale structures is often comparable or stronger than the background magnetic field in the magnetotail. Of course, such mesoscale structures were observed by previous missions. However, now their inner geometry can be investigated with the multipoint analysis.

One of the mesoscale structures actively studied during the last years are dipolarization fronts (DFs) (Nakamura et al., 2009; Runov et al., 2009, 2011; Schmid et al., 2011, and references therein) - a sharp increase of the $B_{\mathrm{Z}}$ component of the magnetic field in the vicinity of the magnetotail neutral plane. This $B_{\mathrm{Z}}$ increase is accompanied by a burst of the elec- tric field with the magnitude of about $\sim 10 \mathrm{mV} \mathrm{m}^{-1}$ (see, e.g. Nakamura et al., 2009; Runov et al., 2011). DFs often propagate towards the Earth. Based on numerical modelling, DFs are associated with the spontaneous magnetic reconnection in the magnetotail (e.g. Sitnov et al., 2009). Geometry and parameters of DFs change only slightly on the course of their Earthward motion (Runov et al., 2009). The shape of a DF is supported by the dawn-dusk ion and electron currents at the front (see Runov et al., 2011; Schmid et al., 2011, and references therein). Therefore, one could assume the existence of two particle populations: trapped (or captured) particles moving with the DF and background plasma.

The role of DFs in particle acceleration is the subject of active investigations. First of all, the rapid increase of $B_{\mathrm{Z}}$ leads to the betatron (or/and Fremi) acceleration of adiabatic electrons (see, e.g. Apatenkov et al., 2007; Fu et al., 2011). Nonadiabatic ions can gain some energy due to the reflection from the DF (Zhou et al., 2010). Magnetic field fluctuations near the front can provide the turbulent ion acceleration (Ono et al., 2009).

DF can be also presented as a moving nonlinear electromagnetic wave. Background ions could interact with such a wave in a resonance manner. It is well known that propagating a wave in the presence of the background magnetic field can capture background particles and accelerate them along the wave front (so called surfactron acceleration, originally proposed by Sagdeev, 1966). This mechanism was considered before for a shock wave (see, e.g. Zank et al., 1996; Simnett et al., 2005, and references therein). For harmonic electromagnetic waves the surfatron mechanism is investigated with the numerical modelling (Takeuchi et al., 1987), in the laboratory experiment (Yugami et al., 1996) and in the frame of the analytical theory (Neishtadt et al., 2009; Artemyev et al., 2010). The non-harmonic wave is considered by Takeuchi $(2005,2008)$, where term 'magnetic trapping' was introduced for the systems with an electromagnetic wave to 

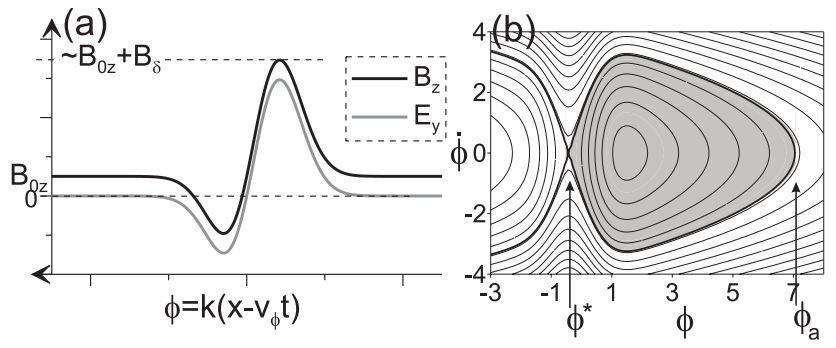

Fig. 1. Panel (a) demonstrates the model magnetic and electric fields. Panel (b) demonstrates the phase portrait of the Hamiltonian $H_{\phi}$, grey colour denotes the oscillation region.

underline the difference with the classical surfatron mechanism proposed initially for an electrostatic wave.

In comparison with other mechanisms of particle acceleration by DF, the considered surfatron mechanism can produce a small population of ions with energy larger than $100 \mathrm{keV}$ under magnetotail conditions. Betatron (and/or Fermi) mechanism (Apatenkov et al., 2007; Fu et al., 2011) can be considered only for adiabatic electrons. Reflection of nonadiabatic ions provides energy gain of a few $\mathrm{keV}$ (Zhou et al., 2010). Electromagnetic fluctuations lead to change in the power law of whole energy spectra (Ono et al., 2009). Therefore, effective acceleration of a small ion population could correspond only to the resonance particle interaction with DF.

In this paper, we study high energy particles observed near DFs. We develop the analytical model of the particle resonance acceleration by a DF and compare the observed data with the theoretical predictions.

\section{Spacecraft data and methods}

We collect the statistics of DFs observed by Interball-tail spacecraft during 1995-1998. Magnetic field data from the ASPI/MIF-M experiment (Klimov et al., 1997) and low energy $(<24 \mathrm{keV})$ ion moments from the CORALL experiment (Yermolaev et al., 1997) are analysed to reveal DFs. High energy particles in the range $20-800 \mathrm{keV}$ are measured with DOK-2 experiment (Lutsenko et al., 1998). DOK-2 has record energy resolution $\sim 7-8 \mathrm{keV}$ and is capable of observing fine structures in energy spectra (see, e.g. Lutsenko and Kudela, 1999). CORALL could underestimate the ion bulk flows along Earth-Sun direction due to the specific configuration. Therefore, all CORALL velocities are confirmed with electron moments, obtained from ELECTRON experiment (Sauvaud et al., 1997).

Between 1995 to 1998 Interball-tail crossed the magnetotail current sheet at the downtail distance of $-30 R_{\mathrm{E}}<x<$ $-11 R_{\mathrm{E}}$. Due to the orbit, the Interball-tail can be inside the plasma sheet up to several hours. During such intervals, DOK-2 often observes the relatively narrow maxima in
Table 1. The list of events.

\begin{tabular}{|c|c|c|c|c|c|}
\hline$N$ & Date & $\begin{array}{l}B_{0 \mathrm{z}} \\
\mathrm{nT}\end{array}$ & $\begin{array}{c}E_{\mathrm{y}}^{*} \\
\mathrm{mV} \mathrm{m}^{-1}\end{array}$ & $\begin{array}{c}W \\
\mathrm{keV}\end{array}$ & $\begin{array}{l}R_{\mathrm{y}} \\
R_{\mathrm{E}}\end{array}$ \\
\hline 1 & 1 Dec 1995: 15:30-15:45 & 5 & 4 & 79 & 3.09 \\
\hline 2 & 3 Nov 1996: $14: 40-14: 50$ & 1 & 2 & 30 & 2.34 \\
\hline 3 & 12 Dec 1996: 13:05-13:25 & 1 & 1.6 & 56 & 5.47 \\
\hline 4 & 12 Dec 1996: 13:57-13:58 & 2 & 7.2 & 136 & 2.95 \\
\hline 5 & 12 Dec 1996: 13:59-14:05 & 2 & 7.2 & 93 & 2.02 \\
\hline 6 & 12 Dec 1996: 14:00-14:10 & 2 & 3.2 & 192 & 9.38 \\
\hline 7 & 12 Dec 1996: 14:10-14:20 & 2 & 4.8 & 158 & 5.14 \\
\hline 8 & 12 Dec 1996: 14:15-14:18 & 3 & 9.6 & 192 & 3.13 \\
\hline 9 & 12 Dec 1996: 14:18-14:19 & 3 & 9.6 & 164 & 2.67 \\
\hline 10 & 12 Dec 1996: 14:20-14:21 & 3 & 9.6 & 184 & 2.99 \\
\hline 11 & 12 Dec 1996: $14: 21-14: 22$ & 3 & 9.6 & 152 & 2.47 \\
\hline 12 & 12 Dec 1996: 15:30-15:50 & 2 & 1.6 & 158 & 15.4 \\
\hline 13 & 12 Dec 1996: 16:30-16:50 & 2 & 1.6 & 93 & 9.08 \\
\hline 14 & 12 Dec 1996: 16:55-17:02 & 2 & 4 & 232 & 9.06 \\
\hline 15 & 12 Dec 1996: 17:03-17:06 & 2 & 4 & 271 & 10.6 \\
\hline 16 & 12 Dec 1996: 17:06-17:10 & 2 & 4 & 63 & 2.46 \\
\hline 17 & 12 Dec 1996: 17:25-17:40 & 2 & 3.2 & 112 & 5.47 \\
\hline 18 & 12 Dec 1996: 17:45-17:50 & 1.5 & 3 & 76 & 4.00 \\
\hline 19 & 12 Dec 1996: 17:52-17:55 & 2 & 1.6 & 80 & 7.81 \\
\hline 20 & 12 Dec 1996: 17:55-17:57 & 2 & 3.2 & 207 & 10.1 \\
\hline 21 & 12 Dec 1996: 17:57-18:00 & 2 & 3.2 & 108 & 5.27 \\
\hline 22 & 12 Dec 1996: 18:05-18:10 & 3 & 7.2 & 192 & 4.17 \\
\hline 23 & 12 Dec 1996: 18:10-18:15 & 3 & 6 & 159 & 4.14 \\
\hline 24 & 12 Dec 1996: 19:15-19:23 & 3 & 4.8 & 100 & 3.26 \\
\hline 25 & 10 Nov 1997: 00:30-00:50 & 5 & 8 & 121 & 2.36 \\
\hline 26 & 10 Nov 1997: 04:10-04:22 & 6 & 7.2 & 71 & 1.54 \\
\hline 27 & 2 Dec 1997: 14:06-14:10 & 2 & 4 & 71 & 2.77 \\
\hline 28 & 2 Dec 1997: 14:40-14:50 & 1 & 5 & 32 & 5.00 \\
\hline 29 & 6 Dec 1997: 07:40-07:55 & 1 & 2 & 86 & 6.72 \\
\hline 30 & 5 Nov 1998: 08:30-08:40 & 4 & 6.4 & 58 & 1.42 \\
\hline 31 & 5 Nov 1998: 09:40-09:50 & 4 & 4.8 & 52 & 1.69 \\
\hline 32 & 5 Nov 1998: 10:15-10:25 & 4 & 8 & 85 & 1.66 \\
\hline 33 & 5 Nov 1998: 11:00-11:10 & 2 & 4.8 & 71 & 2.31 \\
\hline 34 & 5 Nov 1998: 12:30-12:40 & 2 & 2.4 & 71 & 4.62 \\
\hline 35 & 9 Nov 1998: 02:20-02:27 & 2 & 4.8 & 86 & 2.80 \\
\hline 36 & 9 Nov 1998: 02:27-02:32 & 2 & 4 & 121 & 4.72 \\
\hline 37 & 9 Nov 1998: 02:37-02:45 & 5 & 8 & 112 & 2.19 \\
\hline 38 & 9 Nov 1998: 04:05-04:20 & 4 & 8 & 158 & 3.09 \\
\hline 39 & 1 Dec 1998: 12:00-12:10 & 3 & 7.2 & 68 & 1.48 \\
\hline 40 & 1 Dec 1998: 13:05-13:15 & 2 & 4.8 & 116 & 3.78 \\
\hline 41 & 1 Dec 1998: 14:40-14:50 & 2 & 2.4 & 93 & 6.05 \\
\hline 42 & 9 Dec 1998: 07:20-07:40 & 2 & 1.6 & 141 & 13.8 \\
\hline 43 & 12 Dec 1998: 16:50-17:00 & 3 & 1.8 & 96 & 8.33 \\
\hline 44 & 12 Dec 1998: 17:05-17:20 & 3 & 2.4 & 131 & 8.53 \\
\hline 45 & 12 Dec 1998: 18:10-18:25 & 3 & 4.8 & 58 & 1.89 \\
\hline 46 & 12 Dec 1998: 20:45-21:00 & 2.5 & 3 & 147 & 7.66 \\
\hline 47 & 12 Dec 1998: 21:35-21:45 & 2 & 3.2 & 185 & 9.03 \\
\hline 48 & 12 Dec 1998: 21:45-21:50 & 2 & 2.4 & 165 & 10.7 \\
\hline 49 & 20 Dec 1998: 09:45-10:00 & 2 & 2 & 74 & 5.74 \\
\hline 50 & 20 Dec 1998: 11:15-11:35 & 5 & 3 & 117 & 6.10 \\
\hline 51 & 20 Dec 1998: 12:35-12:45 & 2 & 4.8 & 224 & 7.29 \\
\hline
\end{tabular}

the energy spectra close to $\sim 100-200 \mathrm{keV}$ (Lutsenko et al., 2008). We collect the dataset of simultaneous observations of DFs and bursts of energetic ions. All DFs are observed in 
the vicinity of the neutral sheet $\left(B_{\mathrm{X}}\right.$ is small). For each event, we estimate the value of the background magnetic field $B_{0 \mathrm{z}}$ (the value of $B_{\mathrm{Z}}$ in the vicinity of the neutral plane before the $\mathrm{DF})$, the amplitude of the ion bulk velocity $V_{\mathrm{x}}$ during the DF, the corresponding electric field $E_{\mathrm{y}}^{*}=-B_{0 \mathrm{z}} V_{\mathrm{x}} / c$ and the energy $W$ related to the observed maximum of the high energy ion flux (see Fig. 4). The list of DF observations with the main parameters is given in Table 1. Below, we propose the resonance mechanism to describe the ion acceleration up to the energy $\sim 100-300 \mathrm{keV}$ without strong scattering.

\section{Theoretical model of particle acceleration}

This section is devoted to the description of the resonance ion acceleration by the DF. We use a simplified model geometry: the particle motion is described in the approximation of the neutral plane. Only the single component of the magnetic field $B_{\mathrm{Z}}=B_{0 \mathrm{z}}+\delta B_{\mathrm{Z}}(x, t)$ is taken into account. Here $\delta B_{\mathrm{Z}}(x, t)=-B_{\delta} f(\phi)$ corresponds to the DF, argument $\phi=k\left(x-v_{\phi} t\right)$ describes the direction of DF propagation, $1 / k$ and $v_{\phi}$ are the DF thickness and the earthward velocity. Background magnetic field $B_{0 z}$ is assumed to be a constant. This assumption is reasonable if time of particle acceleration is much smaller than $L_{\mathrm{X}} / v_{\phi}$, where $L_{\mathrm{X}}$ is the spatial scale of $B_{0 z}$ variation along the $\mathrm{x}$-axis in the Earth's magnetotail. Below, we demonstrate that this condition is satisfied. The single component of the electric field is $\delta E_{\mathrm{y}}(\phi)=-\frac{v_{\phi}}{c} B_{\delta} f(\phi)$ accordingly to the Maxwell equations $\left(\operatorname{curl}\left(\delta E \boldsymbol{e}_{\mathrm{y}}\right)=-\boldsymbol{e}_{\mathrm{z}} \frac{1}{c} \frac{\partial \delta B}{\partial t}, \operatorname{div}\left(\delta E \boldsymbol{e}_{\mathrm{y}}\right)=0\right)$. We assume $f(\phi)=\phi \exp \left(-\left(\phi+\phi_{0}\right)^{2}\right)$ and introduce the dimensionless parameter $h=B_{\delta} / B_{\mathrm{z} 0}>1$. Figure 1a shows the profiles of $B_{\mathrm{z}}$ and $\delta E_{\mathrm{y}}$ as functions of $\phi$.

In previous publications, the analytical model of the surfatron acceleration was proposed for the similar system, but with $f(\phi)=\sin \phi$ (Takeuchi et al., 1987; Neishtadt et al., 2009) and with $f(\phi)=\tanh \phi$ (Takeuchi, 2005). Here, we consider the profile of magnetic field $\delta B_{\mathrm{Z}}$ close to one described by Takeuchi (2008).

The equations of the nonrelativistic ion motion have the following form (we neglect the motion along z-direction):

$$
\left\{\begin{array}{l}
\dot{u}_{\mathrm{x}}=(1-h f(\phi)) u_{\mathrm{y}} \\
\dot{u}_{\mathrm{y}}=-u_{\phi} h f(\phi)-(1-h f(\phi)) u_{\mathrm{x}}
\end{array}\right.
$$

Here, we introduce the dimensionless variables: $t \rightarrow t \omega_{n}$, $\boldsymbol{u}=\boldsymbol{v} / v_{0}$, particle coordinates $\boldsymbol{r} \rightarrow \boldsymbol{r} \omega_{n} / v_{0}, \kappa=k v_{0} / \omega_{n}$, $u_{\phi}=v_{\phi} / v_{0}$, where $\omega_{n}=q B_{0 z} / m c, q$ is charge and $m$ is mass, $v_{0}$ is the particle thermal velocity. Far from the DF (when $\phi \gg 1$ and $f(\phi) \sim 0$ ) the particle rotates around the Larmor circle: $\dot{u}_{\mathrm{x}}=u_{\mathrm{y}}$ and $\dot{u}_{\mathrm{y}}=-u_{\mathrm{x}}$. If this circle in the velocity space is intersected by the line $u_{\mathrm{x}}=u_{\phi}$ (the condition of the Cherenkov resonance), then the particle can interact with the DF in a resonance manner. For such particle $\dot{\phi}=\kappa\left(u_{\mathrm{x}}-u_{\phi}\right) \sim 0$ and we can rewrite system (1) near the resonance:

$$
\left\{\begin{array}{l}
\ddot{\phi}=\kappa u_{\mathrm{y}}\left(1-h \phi e^{-\left(\phi+\phi_{0}\right)^{2}}\right) \\
\dot{u}_{\mathrm{y}}=-u_{\phi}
\end{array}\right.
$$

Here, we assume that $\kappa \gg 1$ ( $v_{0}$ is large enough) and the variable $\phi$ changes faster than $v_{\mathrm{y}}$. In this case, we can write the Hamiltonian for $\phi: H_{\phi}=\frac{1}{2} \dot{\phi}^{2}+\kappa u_{\mathrm{y}}(\phi-h \Psi(\phi))$, where $\Psi(\phi)=\frac{1}{2} e^{-\left(\phi+\phi_{0}\right)^{2}}+\frac{\sqrt{\pi}}{2} \phi_{0} \operatorname{erf}\left(\phi+\phi_{0}\right)$. The phase portrait of the Hamiltonian $H_{\phi}$ is shown in Fig. $1 \mathrm{~b}$. There are the so called oscillation regions (marked by the grey colour) and the transient regions. A particle captured into the oscillation region moves with the DF ( $\dot{\phi}$ oscillates around zero) and absolute value of its velocity $u_{\mathrm{y}}$ increases according to the second equation in Eq. (2). The boundary of the oscillation region is called a separatrix. If the area bounded by the separatrix, $S$, grows with time then particles from the transient region can be captured inside the oscillation region. The expression for $S$ can be written as (Neishtadt et al., 2009; Artemyev et al., 2010):

$$
\begin{aligned}
& S=\sqrt{2} \oint \sqrt{H_{\phi}-\kappa u_{\mathrm{y}}(\phi-h \Psi(\phi))} d \phi= \\
& \sqrt{2 \kappa\left|u_{\mathrm{y}}\right|} \int_{\phi^{*}}^{\phi_{a}} \sqrt{\left|\phi^{*}-\phi-h\left(\Psi\left(\phi^{*}\right)-\Psi(\phi)\right)\right|} d \phi
\end{aligned}
$$

Here $\phi^{*}$ is the $\phi$ value in the saddle point and $\phi_{a}$ is shown in Fig. 1b. The line $u_{\mathrm{x}}=u_{\phi}$ intersects the Larmor circle in two points (with positive and negative $u_{\mathrm{y}}$ ). However, only if $u_{\mathrm{y}}$ is negative, the area $S \sim \sqrt{\left|u_{\mathrm{y}}\right|} \sim \sqrt{\left|u_{0 \mathrm{y}}-t u_{\phi}\right|}$ grows with time (here $u_{0 \mathrm{y}}$ is a value of $u_{\mathrm{y}}$ just after the capture). Therefore, particles can be captured only in one point. Modelling of the capture is demonstrated in Fig. 2. We integrate system (1) numerically. Initially the particle moves along the Larmor circle far from the DF. Then the DF comes and the particle is captured. The captured particle moves with the DF and is accelerated along the front $u_{\mathrm{y}} \sim-t u_{\phi}$ (in the dimensional form $\left.v_{\mathrm{y}} \sim-v_{\phi} t \omega_{n}\right)$. During the captured motion particle energy $\frac{1}{2}\left(u_{\mathrm{x}}^{2}+u_{\mathrm{y}}^{2}\right)$ grows as $\sim \frac{1}{2}\left(v_{\phi} t\right)^{2}$. Particle motion inside the oscillation region results in periodical oscillations of $u_{\mathrm{y}}$ and $y$ variables also presented in Fig. 2 (see descriptiop of these oscillations in Neishtadt et al., 2009; Artemyev et al., 2010)

\section{Dipolarization fronts and high energy ions}

In this section, we describe four examples of the DF observations by Interball-tail spacecraft. Magnetic field data are presented in Fig. 3. There is the typical increase of the normal component of the magnetic field $B_{\mathrm{Z}}$ during the DF. The zones of DFs are marked by arrows showing also time intervals of observations of high energy ions by DOK-2. The corresponding energy spectra are shown in Fig. 4. The grey 


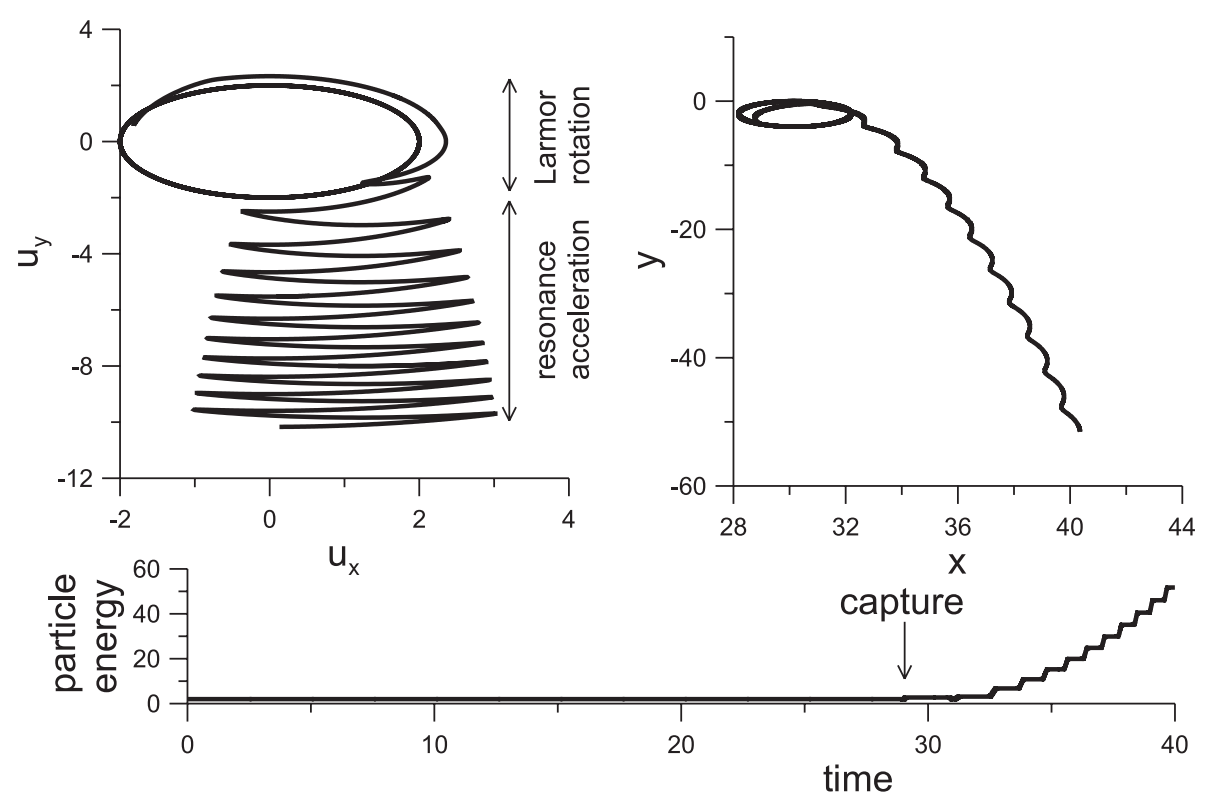

Fig. 2. The resonance particle trajectory is shown in the velocity and coordinate spaces. Also the particle energy $\left(u_{\mathrm{x}}^{2}+u_{\mathrm{y}}^{2}\right) / 2$ as a function of dimensionless time $t \omega_{n}$ is shown.

colour highlights the energy range where the increase of particle flux is observed. For the four observed events, energies are $W \sim 200,150,125$ and $75 \mathrm{keV}$, respectively.

If we consider the observed high energy particles as captured and accelerated population, we can estimate the "hidden" (not measured directly) parameters of DFs. We can derive the expression for the DF width along the dawn-dusk direction, $R_{\mathrm{y}}$.

The energy of captured particles is $\frac{1}{2} m\left(u_{\mathrm{x}}^{2}+u_{\mathrm{y}}^{2}\right) \sim$ $\frac{1}{2} m u_{\mathrm{y}}^{2} \sim \frac{1}{2} u_{\phi}^{2} t^{2}$ and in the dimensional form we have $W=$ $\frac{1}{2} m v_{\phi}^{2}\left(\omega_{n} t\right)^{2}$. The corresponding spatial scale $R_{\mathrm{y}}=\int v_{\mathrm{y}} d t=$ $\frac{1}{2} v_{\phi} \omega_{n} t^{2}$. Therefore, we can write:

$R_{\mathrm{y}}=\frac{c}{v_{\phi}}\left(W / q B_{0 \mathrm{z}}\right)=W /\left|q E_{\mathrm{y}}^{*}\right|$

Thus, one can determine the spatial scale $R_{\mathrm{y}}$ from the estimates of $W$ and $E_{\mathrm{y}}^{*}=-\left(v_{\phi} / c\right) B_{0 \mathrm{z}}$. We assume that the DF velocity, $v_{\phi}$ is equal to bursts bulk flow velocity, $V_{\mathrm{x}}$. For each event from Table 1 , we obtain corresponding $R_{\mathrm{y}}$. The distributions of observed $W$ and estimated $R_{\mathrm{y}}$ are shown in Fig. 5. For the majority of DFs, the observed energies $W$ are distributed in the range $75-200 \mathrm{keV}$. The mean value of the DF width is $\left\langle R_{\mathrm{y}}\right\rangle \approx 6 R_{\mathrm{E}}$.

Corresponding time of particle acceleration $\sim \sqrt{2 R_{\mathrm{y}} / v_{\phi} \omega_{n}}$ is much smaller than $L_{\mathrm{x}} / v_{\phi}$ if

$L_{\mathrm{x}}>\sqrt{2 R_{\mathrm{y}} v_{\phi} / \omega_{n}} \sim 2 R_{\mathrm{E}}$, where $L_{\mathrm{x}}$ is defined above. This condition is almost satisfied for $x \sim-25 R_{\mathrm{E}}$, where our observations were made. Thus, we can consider $B_{0 z}=$ const.

Here, we should notice that the proposed mechanism of ion acceleration can describe the formation of the ion population with energy $\sim 100-300 \mathrm{keV}$ in considered the mag- netic field configuration. However, the width of observed maxima of particle flux in the energy range could be related to some other effect (e.g. time-of-flight effect, see for details Lutsenko et al., 2008).

\section{Discussion}

In this paper, we propose the mechanism responsible for the formation of bursts of energetic ions in the Earth magnetotail based on the resonance interaction of particles with dipolarization fronts. However, we should mention another possible mechanism considered by Lutsenko et al. (2008) for the same type of data. Namely ions can gain energy due to acceleration by electrostatic and inductive electric fields in the course of the so called "current filament disruptions" (local magnetic reconnection). In this case, the increase of $B_{\mathrm{Z}}$ is considered as a temporal event, but not as a spatially localized moving structure. In comparison with the mechanism proposed above, such disruptions correspond to a smaller spatial scale of the acceleration region and a larger value of the electric field. We estimate the electric field to be $\sim 5 \mathrm{mV} \mathrm{m}^{-1}$ and the spatial scale to be $\sim 6 R_{\mathrm{E}}$, while Lutsenko et al. (2008) suggest that the electric field is five times larger and the spatial scale is five times smaller. The relation between these two mechanisms can be further investigated in numerical modelling: it is well known that magnetic reconnection (and corresponding current filament disruption) leads to the formation of the dipolarization front (Sitnov et al., 2009).

We develop the analytical model of the charged particles capture and acceleration by the DF. We assume that DFs propagate in the background plasma and particles from the 
background population can be captured. A particle can be captured if it has the resonance velocity near the DF, but not every resonance particle is captured. The capture is the probabilistic process and the capture probability $\Pi \sim u_{\phi} / \sqrt{\kappa}$ (see Neishtadt et al., 2009; Artemyev et al., 2010, and references therein). The value of $\Pi$ is small due to the large $\kappa$ and only a small part of the resonance population is actually captured.

In this paper, we describe the resonance acceleration only for ions. However, from a theoretical point-of-view electrons could also be captured. For ions the DF is a relatively fast and thin (along $x$ ) structure, while for electrons the DF is a slow (regarding their thermal motion) and thick structure. Therefore, to describe the electron interaction with the DF, another approach should be applied (see, e.g. Neishtadt et al., 2010).

To make all calculations more straightforward, we neglect the influence of $B_{\mathrm{x}}$ component on the ion motion and also assume the planar geometry of the DF. The first simplification is reasonable, because the investigation of the resonance wave-ion interaction in a complete model of a current sheet (with $B_{\mathrm{x}}(z)$ and $B_{0 \mathrm{z}}$ ) reveals the similar effect of capture and acceleration (Vainchtein et al., 2004). The second simplification (neglecting curvature of the DF) could be understood because the detailed spacecraft observations demonstrate the positive curvature of the DF. The DF surface in (x,z)-plane could be approximated by a parabola $x \sim z^{2}$ (Runov et al., 2009). The theoretical model of the wave-particle resonance interaction shows that the particle acceleration is stable and particles do not leave the resonance in such a front curvature (Bulanov and Sakharov, 2000). We also neglect the wave activity near the DF and its influence on the particle acceleration. To support this simplification, we notice that the presence of an additional high-frequency noise could only limit the time of acceleration, but cannot stop the resonance interaction (Artemyev et al., 2010, 2011).

Obtained estimates of $R_{\mathrm{y}}$ in a fortunate case could be verified with the simultaneous observations of the DF by two spacecraft with different positions along the dawn-dusk direction. At 20 December 1998 (events 49 and 50 from the Table 1) Interball-tail was at $(-24,12,-5) R_{\mathrm{E}}$ and Geotail was at $(-14,8,-3) R_{\mathrm{E}}$. Both spacecraft observed the DF with the earthward direction of propagation: Interball-tail observed DFs first and Geotail a few minutes after because of the separation along the Earth-Sun direction (these data are not shown here). Our estimates for these events give $R_{\mathrm{y}} \sim 6 R_{\mathrm{E}}$ and the spacecraft separation along the dawn-dusk direction was about $4 R_{\mathrm{E}}$. Therefore, our estimates do not contradict these two-spacecraft observations.

Due to the low time resolution of CORALL measurements, we could underestimate ion bulk velocity during the DF. Thus, electric field $E_{\mathrm{y}}^{*}$ could also be underestimated, while $R_{\mathrm{y}} \sim 1 / E_{\mathrm{y}}^{*}$ could be overestimated. Unfortunately, the modern spacecraft missions (Cluster, THEMIS) do not have the high resolution energetic particle instrument. Therefore, we cannot apply our technique to data collected by these
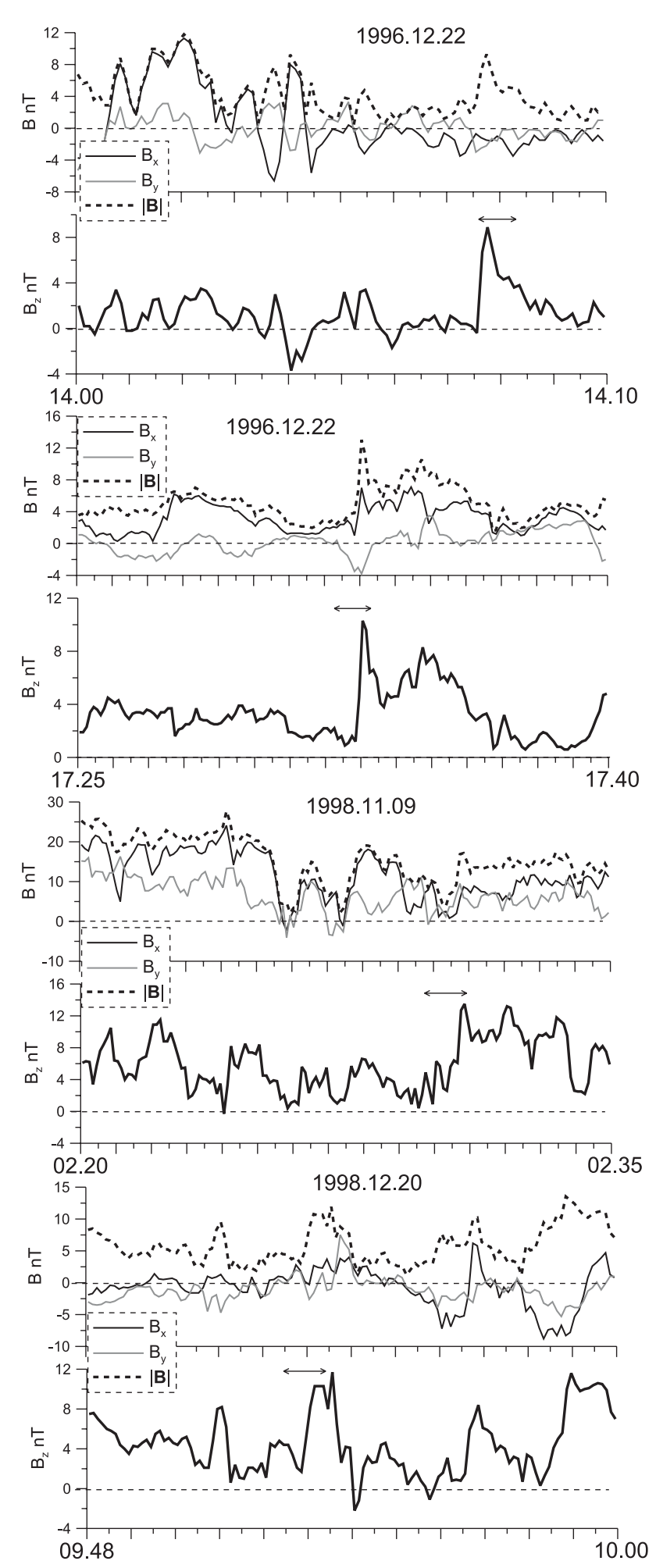

Fig. 3. Four events of DF observations by Interball-tail (x-axis is in UT). The date is given at the top of each panel. 

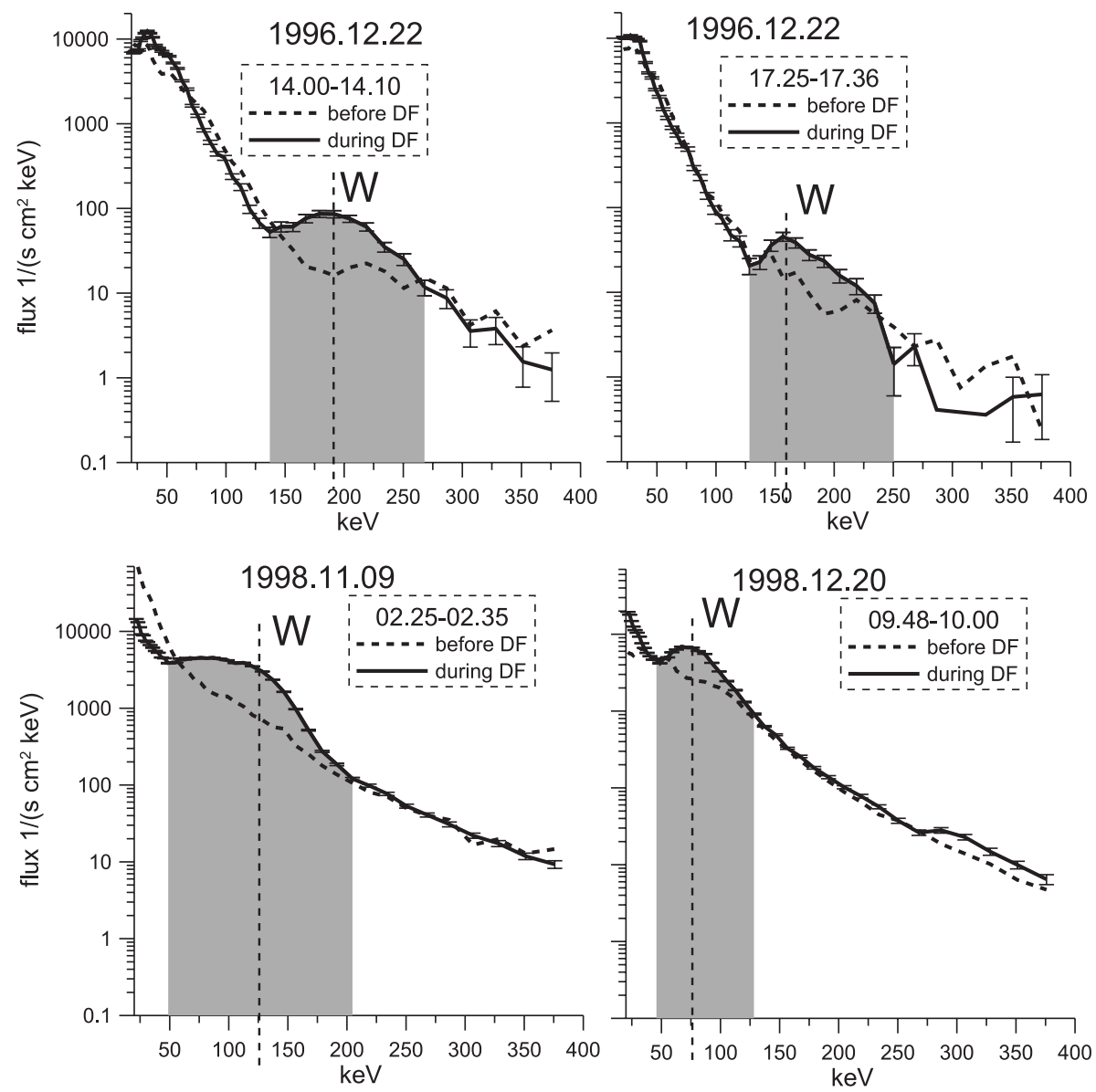

Fig. 4. Ion high-energy spectra for the events from Fig. 3. Bursts of energetic ions are marked by the grey colour.
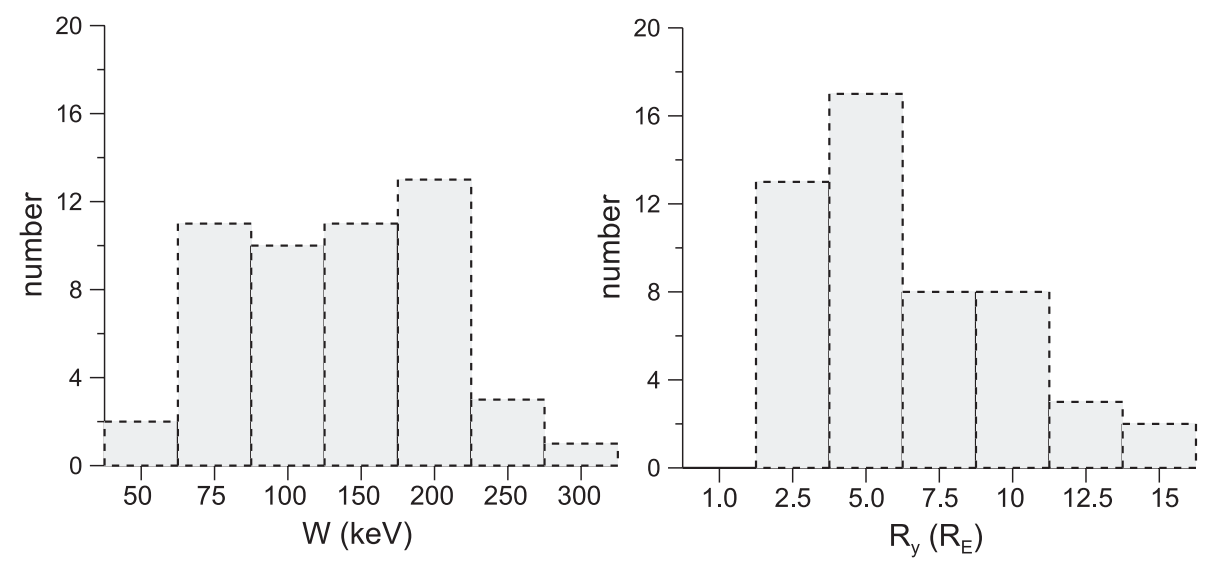

Fig. 5. The distributions of observed values of $W$ and estimates of $R_{\mathrm{y}}$.

missions. However, in the future, we plan to apply our model to study the resonance acceleration in the solar wind, where STEREO-A,B spacecraft also observe the bursts of energetic particles associated with the surfatron acceleration (Klassen et al., 2009).

\section{Conclusions}

In this paper, we study the resonance ion acceleration by DFs with the help of Interball-tail observations and the analytical model. We demonstrate that on the DF the bursts of energetic ions are observed with an average energy of 
about $100-200 \mathrm{keV}$. These high energy populations are described by the model of the resonance acceleration (the surfatron mechanism). We estimate the "hidden" (not measured directly) parameters of the DF by comparison between the model and observations: the width of DFs along the dawndusk direction is found to be $\sim 6 R_{\mathrm{E}}$. The developed approach demonstrates that the combination of the analytical models and the observations of high-energy particle distributions could be a useful instrument for the investigation of mesoscale structures.

Acknowledgements. The authors are grateful to A. I. Neishatdt and A. A. Vasiliev for fruitful discussions and to both Reviewers for useful suggestions and comments. Authors would like to acknowledge Interball instrument teams ASPI/MIF-M, CORALL, ELECTRON and DOK-2 for excellent data. This work was supported by the Russian Foundation for Basic Research (projects no. 10-02-93114, 10-02-00135) and by the European Union Seventh Framework Programme [FP7/2007-2013] under grant agreement no. 269198 Geoplasmas (Marie Curie International Research Staff Exchange Scheme).

Topical Editor R. Nakamura thanks two anonymous referees for their help in evaluating this paper.

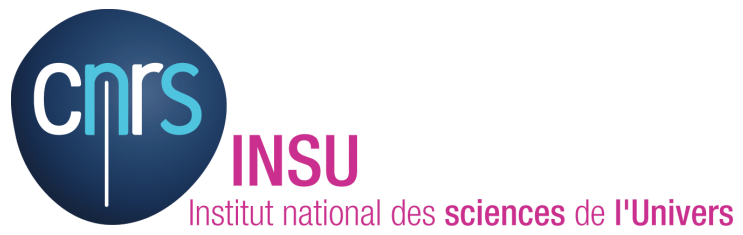

The publication of this article is financed by CNRS-INSU.

\section{References}

Apatenkov, S. V., Sergeev, V. A., Kubyshkina, M. V., Nakamura, R., Baumjohann, W., Runov, A., Alexeev, I., Fazakerley, A., Frey, H., Muhlbachler, S., Daly, P. W., Sauvaud, J.-A., Ganushkina, N., Pulkkinen, T., Reeves, G. D., and Khotyaintsev, Y.: Multi-spacecraft observation of plasma dipolarization/injection in the inner magnetosphere, Ann. Geophys., 25, 801-814, doi:10.5194/angeo-25-801-2007, 2007.

Artemyev, A. V., Neishtadt, A. I., Zelenyi, L. M., and Vainchtein, D. L.: Adiabatic description of capture into resonance and surfatron acceleration of charged particles by electromagnetic waves, Chaos, 20, 043128, doi:10.1063/1.3518360, 2010.

Artemyev, A., Vainchtein, D., Neishtadt, A., and Zelenyi, L.: Resonant acceleration of charged particles in the presence of random fluctuations, Phys. Rev. E, 84, 046213, doi:10.1103/PhysRevE.84.046213, 2011.

Bulanov, S. V. and Sakharov, A. S.: Effect of the Magnetic Field on the Resonant Particle Acceleration, Plasma Physics Reports, 26, 1005-1014, doi:10.1134/1.1331136, 2000.

Fu, H. S., Khotyaintsev, Y. V., André, M., and Vaivads, A.: Fermi and betatron acceleration of suprathermal electrons behind dipolarization fronts, Geophys. Res. Lett., 381, L16104, doi:10.1029/2011GL048528, 2011.

Klassen, A., Gómez-Herrero, R., Müller-Mellin, R., Böttcher, S., Heber, B., Wimmer-Schweingruber, R., and Mason, G. M.:
STEREO/SEPT observations of upstream particle events: almost monoenergetic ion beams, Ann. Geophys., 27, 2077-2085, doi:10.5194/angeo-27-2077-2009, 2009.

Klimov, S., Romanov, S., Amata, E., Blecki, J., Büchner, J., Juchniewicz, J., Rustenbach, J., Triska, P., Woolliscroft, L. J. C., Savin, S., Afanas'yev, Yu., de Angelis, U., Auster, U., Bellucci, G., Best, A., Farnik, F., Formisano, V., Gough, P., Grard, R., Grushin, V., Haerendel, G., Ivchenko, V., Korepanov, V., Lehmann, H., Nikutowski, B., Nozdrachev, M., Orsini, S., Parrot, M., Petrukovich, A., Rauch, J. L., Sauer, K., Skalsky, A., Slominski, J., Trotignon, J. G., Vojta, J., and Wronowski, R.: ASPI experiment: measurements of fields and waves on board the INTERBALL-1 spacecraft, Ann. Geophys., 15, 514-527, doi:10.1007/s00585-997-0514-3, 1997.

Lutsenko, V. N. and Kudela, K.: Almost monoenergetic ions near the Earth's magnetosphere boundaries, Geophys. Res. Lett., 26, 413-416, doi:10.1029/1999GL900002, 1999.

Lutsenko, V. N., Kudela, K., and Sarris, E. T.: The DOK-2 Experiment to Study Energetic Particles by the Tail Probe and Auroral Probe Satellites in the INTERBALL Project, Cosmic Research, 36, 98-107, 1998.

Lutsenko, V. N., Gavrilova, E. A., and Grechko, T. V.: Statistics of fine dispersion structures events in energetic particle spectra: their origin and role in the outer magnetosphere, Ann. Geophys., 26, 2097-2110, doi:10.5194/angeo-26-2097-2008, 2008.

Nakamura, R., Retinò, A., Baumjohann, W., Volwerk, M., Erkaev, N., Klecker, B., Lucek, E. A., Dandouras, I., André, M., and Khotyaintsev, Y.: Evolution of dipolarization in the nearEarth current sheet induced by Earthward rapid flux transport, Ann. Geophys., 27, 1743-1754, doi:10.5194/angeo-27-17432009, 2009.

Neishtadt, A. I., Artemyev, A. V., Zelenyi, L. M., and Vainshtein, D. L.: Surfatron acceleration in electromagnetic waves with a low phase velocity, JETP Letters, 89, 441-447, doi:10.1134/S0021364009090045, 2009.

Neishtadt, A. I., Artemyev, A. V., and Zelenyi, L. M.: Regular and chaotic charged particle dynamics in low frequency waves and role of separatrix crossings, Regular and Chaotic Dynamics, 15, 564-574, doi:10.1134/S1560354710040118, 2010.

Ono, Y., Nosé, M., Christon, S. P., and Lui, A. T. Y.: The role of magnetic field fluctuations in nonadiabatic acceleration of ions during dipolarization, J. Geophys. Res., 114, 5209, doi:10.1029/2008JA013918, 2009.

Runov, A., Angelopoulos, V., Sitnov, M. I., Sergeev, V. A., Bonnell, J., McFadden, J. P., Larson, D., Glassmeier, K.H., and Auster, U.: THEMIS observations of an earthwardpropagating dipolarization front, Geophys. Res. Lett., 36, L14106, doi:10.1029/2009GL038980, 2009.

Runov, A., Angelopoulos, V., Zhou, X.-Z., Zhang, X.-J., Li, S., Plaschke, F., and Bonnell, J.: A THEMIS multicase study of dipolarization fronts in the magnetotail plasma sheet, J. Geophys. Res., 116, A05216, doi:10.1029/2010JA016316, 2011.

Sagdeev, R. Z.: Reviews of Plasma Physics, vol. 4, Consultants Bureau, New York, 1st edn., 1966.

Sauvaud, J.-A., Koperski, P., Beutier, T., Barthe, H., Aoustin, C., Thocaven, J. J., Rouzaud, J., Penou, E., Vaisberg, O., and Borodkova, N.: The INTERBALL-Tail ELECTRON experiment: initial results on the low-latitude boundary layer of the dawn magnetosphere, Ann. Geophys., 15, 587-595, doi:10.1007/s00585- 
997-0587-z, 1997.

Schmid, D., Volwerk, M., Nakamura, R., Baumjohann, W., and Heyn, M.: A statistical and event study of magnetotail dipolarization fronts, Ann. Geophys., 29, 1537-1547, doi:10.5194/angeo29-1537-2011, 2011.

Sharma, A. S., Nakamura, R., Runov, A., Grigorenko, E. E., Hasegawa, H., Hoshino, M., Louarn, P., Owen, C. J., Petrukovich, A., Sauvaud, J.-A., Semenov, V. S., Sergeev, V. A., Slavin, J. A., Sonnerup, B. U. Ö., Zelenyi, L. M., Fruit, G., Haaland, S., Malova, H., and Snekvik, K.: Transient and localized processes in the magnetotail: a review, Ann. Geophys., 26, 9551006, doi:10.5194/angeo-26-955-2008, 2008.

Simnett, G. M., Sakai, J.-I., and Forsyth, R. J.: Proton and electron acceleration by quasi-perpendicular fast magnetosonic shocks in interplanetary space, Astronomy and Astrophysics, 440, 759766, doi:10.1051/0004-6361:20040229, 2005.

Sitnov, M. I., Swisdak, M., and Divin, A. V.: Dipolarization fronts as a signature of transient reconnection in the magnetotail, J. Geophys. Res., 114, A04202, doi:10.1029/2008JA013980, 2009.

Takeuchi, S.: New particle accelerations by magnetized plasma shock waves, Physics of Plasmas, 12, 102901, doi:10.1063/1.2080520, 2005.

Takeuchi, S.: Magnetic Trapping Acceleration in Interplanetary Plasmas, in: International Cosmic Ray Conference, vol. 2 of International Cosmic Ray Conference, pp. 243-246, 2008.
Takeuchi, S., Sakai, K., Matsumoto, M., and Sugihara, R.: Unlimited acceleration of a charged particle by an electromagnetic wave with a purely transverse electric field, Physics Letters A, 122, 257-261, doi:10.1016/0375-9601(87)90818-8, 1987.

Vainchtein, D. L., Rovinsky, E. V., Zelenyi, L. M., and Neishtadt, A. I.: Resonances and Particle Stochastization in Nonhomogeneous Electromagnetic Fields, J. NonLinear Sci., 14, 173-205, doi:10.1007/s00332-003-0576-7, 2004.

Yermolaev, Yu. I., Fedorov, A. O., Vaisberg, O. L., Balebanov, V. M., Obod, Yu. A., Jimenez, R., Fleites, J., Llera, L., and Omelchenko, A. N.: Ion distribution dynamics near the Earth's bow shock: first measurements with the 2D ion energy spectrometer CORALL on the INTERBALL/Tail-probe satellite, Ann. Geophys., 15, 533-541, doi:10.1007/s00585-997-0533-0, 1997.

Yugami, N., Kikuta, K., and Nishida, Y.: Electron Acceleration by a Transverse Electromagnetic Wave Supplemented with a Crossed Static Magnetic Field, Phys. Rev. Lett., 76, 1635-1638, doi:10.1103/PhysRevLett.76.1635, 1996.

Zank, G. P., Pauls, H. L., Cairns, I. H., and Webb, G. M.: Interstellar pickup ions and quasi-perpendicular shocks: Implications for the termination shock and interplanetary shocks, J. Geophys. Res., 101, 457-478, doi:10.1029/95JA02860, 1996.

Zhou, X., Angelopoulos, V., Sergeev, V. A., and Runov, A.: Accelerated ions ahead of earthward propagating dipolarization fronts, J. Geophys. Res., 115, A00I03, doi:10.1029/2010JA015481, 2010 . 